\title{
Performance Evaluation of Error-Correcting Scheme Without Redundancy Code for Noncoherent Chaos Communications
}

\author{
Shintaro ARAI*, Yoshifumi NISHIO ${ }^{\dagger}$, Takaya YAMAZATO ${ }^{\ddagger}$ and Shinji OZAWA* \\ *ITS Laboratory, Faculty of Engineering, Aichi University of Technology \\ 50-2 Manori, Nishihasama-cho Gamagori, Aichi 443-0047 JAPAN \\ \{arai-its, ozawa\}@aut.ac.jp \\ ${ }^{\dagger}$ Department of Electrical and Electronic Engineering, Tokushima University \\ 2-1 Minami-Josanjima, Tokushima, 770-8506 JAPAN \\ nishio@ee.tokushima-u.ac.jp \\ ${ }^{\ddagger}$ EcoTopia Science Institute, Nagoya University \\ C3-1, Furo-cho, Chikusa-ku, Nagoya 464-8063 JAPAN \\ yamazato@nuee.nagoya-u.ac.jp
}

\begin{abstract}
This paper considers a novel error-correcting scheme exploiting chaotic dynamics for noncoherent chaos communication. In our proposed system, two successive chaotic sequences are generated from the same chaotic map; the second sequence is generated with an initial value which is the last value of the first sequence. In this case, successive chaotic sequences having the same chaotic dynamics are created. This feature gives the receiver additional information to correctly recover the information data and thus improves the bit error performance of the receiver. Further, enhanced efficiency also comes from operating on successively modulated data; by involving less redundancy in the error correction system, it can be designed with high coding rate. In this paper, we analyze the scheme's capability, by examining computational times and accuracy rates of error correction. bounds on its capability.
\end{abstract}

\section{INTRODUCTION}

Chaos communication systems are an interesting topic in the field of engineering chaos [1]- [7]. Especially, many researchers focused on the development of noncoherent detection systems which are demodulation techniques using signals modulated by chaos only or chaotic systems. Differential chaos shift keying (DCSK) [1] and the optimal receiver [2] are well-known typical noncoherent systems. Moreover, it is also important to develop a suboptimal receiver with performance equivalent to or similar to the optimal receiver using more efficient algorithms [3] [9].

In our previous research, we focused on the chaotic dynamics and proposed the error-correcting method using the chaotic dynamics [8] [10]. In standard noncoherent chaos communication systems, a binary data bit is modulated in a transmitter by a chaotic sequence of chosen length; the bit is demodulated at the receiver using the modulated sequence and the transmitted unmodulated corresponding sequence. The demodulation of each bit is performed only with the chaotic sequence associated with that bit. We have paid attention to the successive chaotic sequences which are scarcely used for the demodulation in standard chaos communications.

In our proposed system, two successive chaotic sequences are generated from the same chaotic map; the second sequence is generated with an initial value which is the last value of the first sequence. In this case, successive chaotic sequences hav- ing the same chaotic dynamics are created. This feature gives the receiver additional information to correctly recover the information data and thus improves the bit error performance of the receiver. Further, enhanced efficiency also comes from operating on successively modulated data; by involving less redundancy in the error correction system, it can be designed with high coding rate. Preliminary computer simulations have confirmed that the advantage gained in BER performance is about 2-3 dB compared to conventional noncoherent systems. However, the performances of their error-correcting scheme has been only confirmed in primary simulations. In this paper, we analyze the scheme's capability, by examining computational times and accuracy rates of error correction.

\section{SYSTEM OVERVIEW}

We consider the discrete-time binary CSK communication system with the error correcting, as shown in Fig. 1. Detail of each block is described below.

\section{A. Transmitter}

In the transmitter, binary data are encoded by chaotic sequences generated by a chaotic map. In this study, we use a skew tent map which is one of simple chaotic maps, and it is described by Eq. (1)

$$
x_{i+1}= \begin{cases}\frac{2 x_{i}+1-a}{1+a} & \left(-1 \leq x_{i} \leq a\right) \\ \frac{-2 x_{i}+1+a}{1-a} & \left(a<x_{i} \leq 1\right)\end{cases}
$$

where $a$ denotes a position of the top of the skew tent map. Our encoder is designed based on Chaos Shift Keying (CSK) which is a digital modulation system using chaos. Figure 2 shows our encoder for our error-correcting scheme. To perform the error correction at the receiver, $K$ information bit are transmitted as $K$ signal blocks $(0,1, \cdots, j, \cdots, K-1)$. The encoder selects a chaotic signal generator according to the symbol. If the symbol " 1 " is sent, Eq. (1) is used, and if " 0 " is sent, the reversed function of Eq. (1) is used. Thus, the signal vector 


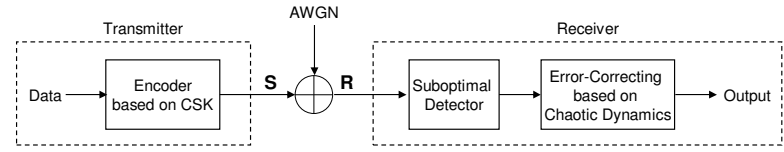

Fig. 1. Block Diagram of Discrete-Time Binary CSK Communication System.

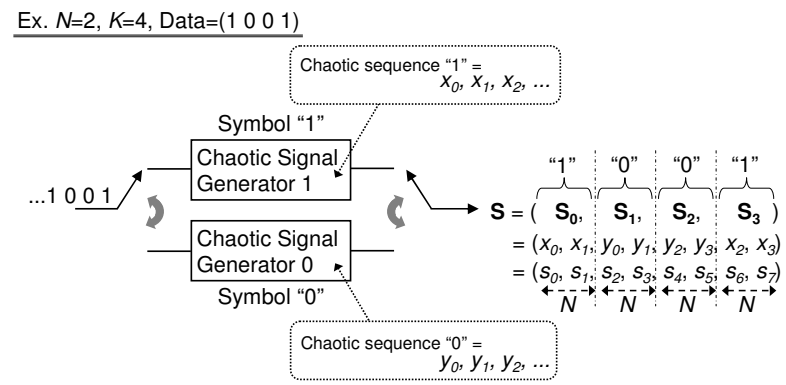

Fig. 2. Encoder based on CSK for error correction.

$\mathbf{S}_{\mathrm{j}}$ is different for each symbol.

[When the symbol " 1 " is sent]

$$
\mathbf{S}_{\mathbf{j}}=\left(x_{\alpha}, f^{(1)}\left(x_{\alpha}\right), \cdots, f^{(i)}\left(x_{\alpha}\right), \cdots, f^{(N-1)}\left(x_{\alpha}\right)\right)
$$

[When the symbol " 0 " is sent]

$$
\mathbf{S}_{\mathbf{j}}=\left(y_{\alpha}, g^{(1)}\left(y_{\alpha}\right), \cdots, g^{(i)}\left(y_{\alpha}\right), \cdots, g^{(N-1)}\left(y_{\alpha}\right)\right)
$$

where $f^{(i)}$ and $g^{(i)}$ are the function of the skew tent map for symbol "1" and " 0 ", respectively, $i$ is the iteration of $f$ or $g, \alpha=N \times j, x_{j}$ or $y_{j}$ denotes the initial value of the $j$ th symbol = " 1 " or " 0 " respectively, $N$ is the chaotic sequence length per 1 bit. When $K$ bit data is transmitted, the length of the data becomes $K \times N$. An initial value is chosen at random when beginning to make signal blocks and is different in each chaotic signal generator. In addition, the $j$-th sequence is generated from the initial value which is the end value of the former sequence with same symbol of $j$-th bit. As an example, we assume $N=2, K=4$ and the data are $(1,0,0,1)$ shown in Fig. 2. In this case, the transmitted signal vector $\mathbf{S}$ is given as follows.

$$
\begin{aligned}
\mathbf{S} & =\left(\mathbf{S}_{\mathbf{0}}, \mathbf{S}_{\mathbf{1}}, \mathbf{S}_{\mathbf{2}}, \mathbf{S}_{\mathbf{3}}\right) \\
& =\left(x_{0}, x_{1}, y_{0}, y_{1}, y_{2}, y_{3}, x_{2}, x_{3}\right) \\
& =\left(s_{0}, s_{1}, s_{2}, s_{3}, s_{4}, s_{5}, s_{6}, s_{7}\right)
\end{aligned}
$$

As one can see, the initial value of the 4th symbol and 3rd symbol is generated by the end value of 1 st symbol and 2 nd symbol, respectively.

\section{B. Channel and noise}

The channel distorts the signal and corrupts it by noise. In this study, noise of the channel is assumed to be the additive white Gaussian noise (AWGN). Thus, the received signals block is given by $\mathbf{R}=\left(r_{0}, r_{1}, \cdots, r_{K N-1}\right)=\mathbf{S}+A W G N$.

\section{Receiver}

The receiver recovers the transmitted signals from the received signals and demodulates the information symbol. Also, the receiver performs the error correction in this study. Since we consider a noncoherent receiver, the receiver memorizes the chaotic map used for the modulation at the transmitter.

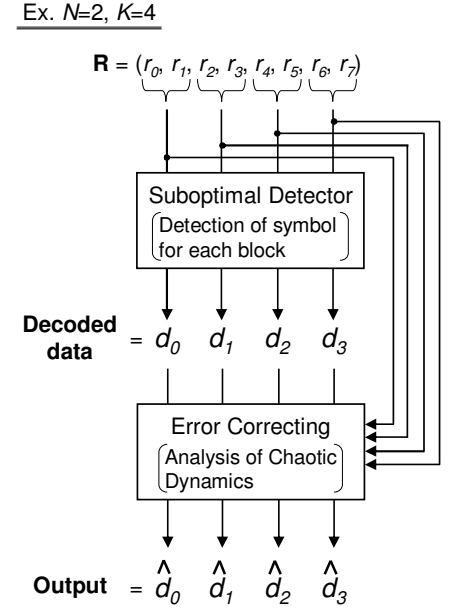

Fig. 3. Operation of proposed error-correcting method.

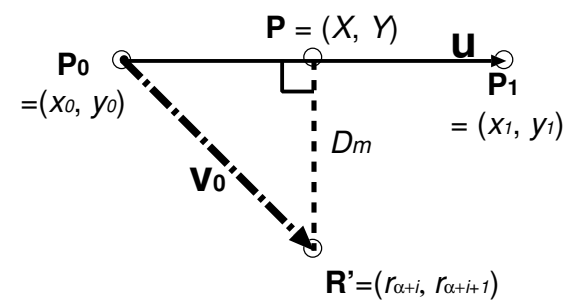

Fig. 4. Calculation of shortest distance.

However, the receiver never knows the initial value of chaos in the transmitter. Our proposed error-correcting method consists of the suboptimal detector and the error correction based on chaotic dynamics, as shown in Fig. 3.

1) Suboptimal detector: First of all, the receiver performs the noncoherent detection for each received block and demodulates each symbol. In this study, we apply the suboptimal detection algorithm of Ref. [9] as the noncoherent detection.

Our suboptimal detector calculates the shortest distance between the received signals and the chaotic map in the $N_{d}$-dimensional space using $N_{d}$ successive received signals $\left(N_{d}: 2,3, \cdots\right)$. As an example, we explain the case of $N_{d}=2$. In this case, we consider two successive signal samples $\mathbf{R}^{\prime}=\left(r_{\alpha+i}, r_{\alpha+i+1}\right)$ as coordinate of chaotic map. To decide which map is closer to the point $\mathbf{R}^{\prime}$ the shortest distance between the point and the map has to be calculated. Therefore, the receiver can calculate the shortest distance using the scalar product of the vector.

Any two points $\mathbf{P}_{\mathbf{0}}=\left(X_{0}, Y_{0}\right)$ and $\mathbf{P}_{\mathbf{1}}=\left(X_{1}, Y_{1}\right)$ are chosen from each straight line in the $N_{d}$-dimensional space, as shown in Fig. 4. Using Fig. 4, the detector can calculate the point $\mathbf{P}=(X, Y)$ closest to $\mathbf{R}$ and the shortest distance $D$ using the following equations.

$$
\begin{aligned}
& \mathbf{P}=(X, Y)=\left(\mathbf{u} \cdot \mathbf{v}_{\mathbf{0}}\right) \mathbf{u}+\mathbf{P}_{\mathbf{0}} \\
D & =\left\|\mathbf{P}-\mathbf{R}^{\prime}\right\| \\
& =\sqrt{\left(X-r_{\alpha+i}\right)^{2}+\left(Y-r_{\alpha+i+1}\right)^{2}}
\end{aligned}
$$

where

$$
\begin{aligned}
\text { unit vector } \mathbf{u} & =\frac{\mathbf{P}_{\mathbf{1}}-\mathbf{P}_{\mathbf{0}}}{\left\|\mathbf{P}_{\mathbf{1}}-\mathbf{P}_{\mathbf{0}}\right\|} \\
\mathbf{v}_{\mathbf{0}} & =\mathbf{R}^{\prime}-\mathbf{P}_{\mathbf{0}} .
\end{aligned}
$$




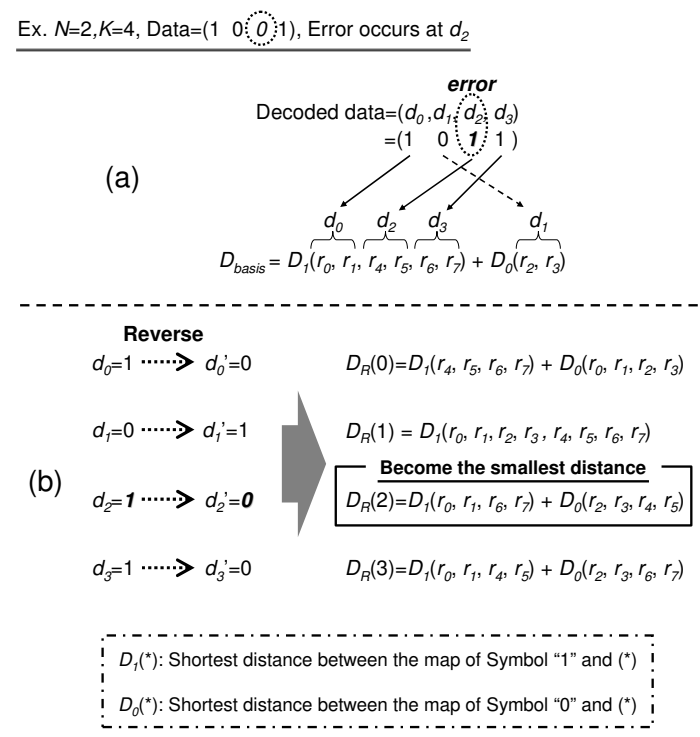

Fig. 5. Analysis of Chaotic Dynamics using Suboptimal Detection.

In the case of 2-dimensional space, there are two straight lines in the space. Therefore, the minimum value in four distances is chosen as the shortest distance $D_{1}$ for symbol " 1 ". In the same way, $D$ of symbol " 0 " is chosen as $D_{0}$. We perform these operations until the last sample (i.e., $r_{\alpha+N-1}$ ) is included, and find their summations $\sum D_{1}$ and $\sum D_{0}$. Finally, we decide the decoded symbol as 1 (or 0 ) for $\sum D_{1}<\sum D_{0}$ (or $\sum D_{1}>\sum D_{0}$ ). The calculation of the shortest distance can be extended to $N_{d}$-dimensional space for $N_{d} \geq 4$.

2) Error-correcting method without Redundancy code: After demodulation of each symbol, the receiver performs the error-correcting method. For error correction without the redundancy code, the receiver uses the received signal samples again. For ease of explanation, we use Fig. 5 and explain an operation of the proposed error-correcting scheme. Here, we use same assumption in the explanation of the encoder (Fig. 2). Also, we assume that decoded symbols become $(1,0,0,1)$ and the detection error has occurred at the 2 nd symbol $\left(d_{2}\right)$, namely the case of 1 bit correction.

First of all, the receiver sorts the received signal samples based on decoded symbols and analyzes the chaotic dynamics of two sequences which are sorted according to decoded symbols, as shown in Fig. 5(a). If the receiver can detects symbols and sorts blocks correctly, we can obtain two successive chaotic sequences based on the chaotic dynamics. However, if the detection error is occurred when the receiver detects symbols, the sorted sequence mixes two chaotic sequences which differ in the chaotic dynamics. We focus on these characteristics of chaos for the error-correcting. For analyzing the chaotic dynamics, the receiver apply our suboptimal detection algorithm, i.e., the calculation of the shortest distance between the chaotic maps and two sorted received sequences. Thus, we define a reference distance $D_{\text {basis }}$ as follows.

$$
\begin{aligned}
D_{\text {basis }} & =D_{1}(\text { Sequence of decoded symbol "1") } \\
& +D_{0} \text { (Sequence of decoded symbol "0") }
\end{aligned}
$$

where $D_{1}($.$) and D_{0}($.$) mean the shortest distance between$ the chaotic map of Symbol "1" and "0", respectively.

Next, we calculate the distance $D_{R}(j)$ for comparing with $D_{\text {basis }}$, where the subscript $R$ means initial character of "Reverse". This equation means the shortest distance between sorted sequence when the $j$-th decoded symbol is reversed and the chaotic map corresponding to their sequences. Namely, we assume the detection error is occurred at $j$-th symbol and calculate $D_{R}(j)$. If the receiver can detects symbols and sorts blocks correctly, $D_{R}(j)$ becomes lager values as compared with $D_{\text {basis. }}$. On the other hands, if the detection error is occurred, some of $D_{R}(0)-D_{R}(K-1)$ become smaller as compared with $D_{\text {basis }}$. The reason for changing the values is also to change combinations of the chaotic dynamics. Therefore, the receiver selects the smallest distance from $D_{\text {basis }}$ and $D_{R}(j)$ and corrects an error.

For instance, Fig. 5(b) shows conceivable combinations of sorted sequences and calculates $D_{R}(j)$ when the detection error has occurred at the 2 nd symbol. In this case, $D_{R}(2)$ becomes the smallest distance as compared with $D_{\text {basis }}$ and other $D_{R}(j)$. Thus, the receiver can determine that the detection error is occurred at the 2 nd symbol.

Although we consider the case of 1 bit correction in this assumption, the case of 2 or more bit can be also performed in the same way. In this case, since the number of the symbols reversed at once, namely the number of conceivable combinations increases, a computation time also increases.

\section{EVALUATION OF PROPOSED ERROR-CORRECTING METHOD}

In this section, we evaluate the performance of the proposed error-correcting method. We carry out computer simulations and investigate three evaluations a bit error rate (BER), an accuracy rate of each bit correction and a computation time. The simulation conditions are as follows. In the transmitting side, we assume $K=32,64$. The parameter of the skew tent map is fixed as $a=0.05$. The chaotic sequence length per 1 bit is $N=4$. For calculation of the shortest distance, we use 4-dimensional space, namely $N_{d}=N=4$. Based on these conditions, we iterate the simulation 10,000 times and calculate BER, the accuracy rate and the computation time.

Figures 6(a) and (b) show the BERs versus $E_{b} / N_{0}$ for $K=32$ and $K=64$, respectively. We plot the performance of the proposed error-correcting method and the performance of the conventional method, namely, the performance without the error-correcting method in Figs. 6(a) and (b). From these figures, we can confirm that the advantage gained in BER performance of the proposed error-correcting method is about 1-2 dB compared to conventional method. However, the performance of 2 bit correction by using the proposed errorcorrecting method is only slightly better than that of its 1 bit correction.

For investigation in detail, we observe the accuracy rate of each bit error correction in Figs. 7(a) and (b). In this figure, the left vertical axis is a percentage of the number of each bit error, the right vertical axis is the accuracy rate of error correction. In general, it is well-known that the percentage of 1 bit error is statistically larger than that of 2 bit error as shown in Figs. 7(a) and (b). Thus, the effect of 1 bit correction by using the proposed error-correcting method on BER performance is also larger than that of its 2 bit correction. However, we can observe that the accuracy rate of its 2 bit correction is better than that 


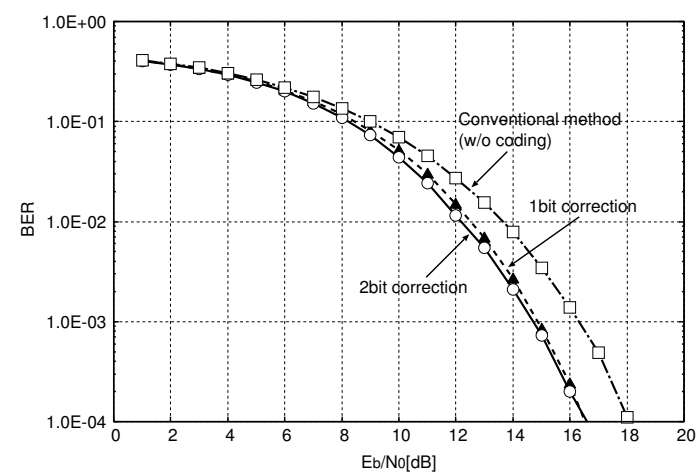

(a) $K=32$.

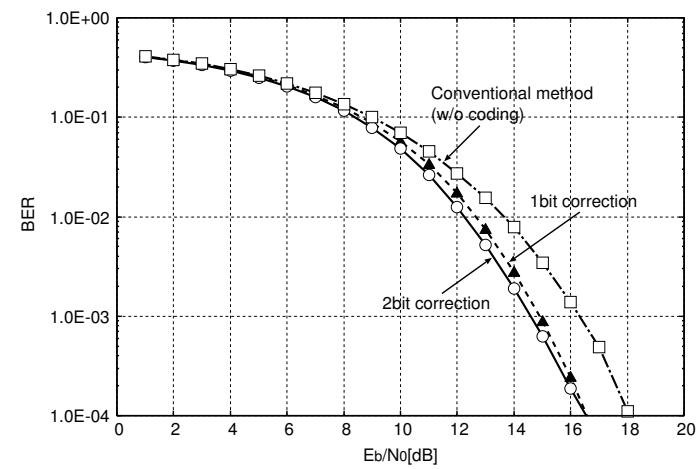

(b) $K=64$.

Fig. 6. BER performance.

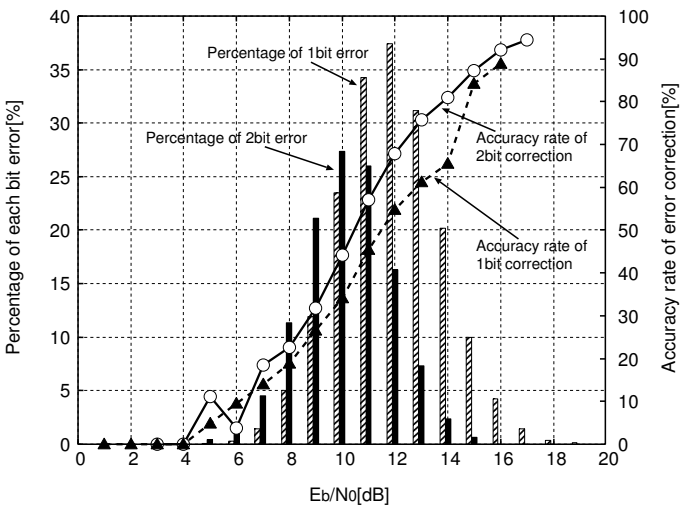

(a) $K=32$.

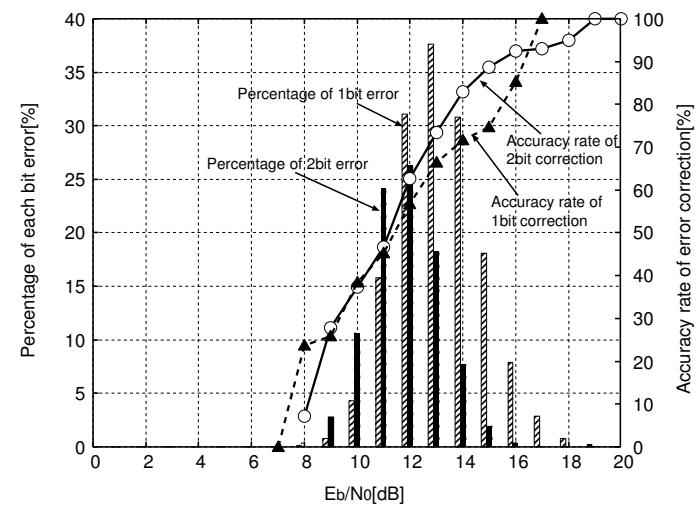

(b) $K=64$.
TABLE I

COMPUTATION TIME FOR ERROR CORRECTION ( $10^{4}$ SYMBOLS).

\begin{tabular}{c|c|c}
\hline \multirow{2}{*}{} & \multicolumn{2}{|c}{ Computation time [sec] } \\
\cline { 2 - 3 } & $K=32$ & $K=64$ \\
\hline w/o coding & 1.88 & 5.15 \\
\hline 1bit correction & 101.7 & 434.4 \\
\hline 2bit correction & 1516.7 & 12264.2
\end{tabular}

of its 1 bit correction from these figures. Namely, it can be said that the 2 bit error correction by using the proposed errorcorrecting method is performed at high accuracy rate although the performance of its 2 bit correction is only slightly better than that of its 1 bit correction.

Finally, we summarize computation times of the proposed error-correcting method in Table I. We carry out three simulations the conventional method (w/o coding), the 1 and 2 bit corrections by using the proposed error-correcting method with same conditions for calculating BER, and calculate a computation time required to decode all the symbols with any fixed $E_{b} / N_{0}$. These simulations are carried out using a PC with a Core2Duo $2.4 \mathrm{GHz} \mathrm{CPU}$ and $2 \mathrm{~GB}$ of RAM. From this table, it can be confirmed that the computational time required the 2 bit correction is largest in the results. Thus, we consider that it is important to simplify the algorithm of the proposed error-correcting method and to reduce extra calculation for improving the computation times.

\section{CONCLUSIONS}

In this study, we have analyzed the proposed errorcorrection's capability, by examining the computational time and the accuracy rate of error correction. As results of computer simulations, it can be said that the 1 bit correction of the proposed error-correcting method is currently more accessible than that of its 2 bit correction, although simplifying the algorithm of the proposed error-correcting method and reducing its extra calculation for improving the computation times are our future works.

\section{REFERENCES}

[1] G. Kolumbán, B. Vizvári, W. Schwarz, and A. Abel, "Differential chaos shift keying: A robust coding for chaos communication," Proc. NDES'96, pp. 87-92, Jun. 1996.

[2] M. Hasler and T. Schimming, "Chaos communication over noisy channels," Int. J. Bifurcation and Chaos, vol. 10, no. 4, pp. 719-736, Apr. 2000.

[3] M. Hasler and T. Schimming, "Optimal and suboptimal chaos receivers," Proc. IEEE, vol. 90, Issue 5, pp. 733-746, May 2002.

[4] F. C. M. Lau and C. K. Tse, Chaos-Based Digital Communication Systems, Springer, 2003.

[5] F. C. M. Lau and C. K. Tse, "On optimal detection of noncoherent chaosshift-keying signals in a noisy environment,” Int. J. Bifurcation and Chaos Vol. 13, pp. 1587-1597, 2003.

[6] W. M. Tam, F. C. M. Lau and C. K. Tse, "Generalized correlation-delayShift-Keying Scheme for Noncoherent Chaos-Based Communication Systems," IEEE Trans. Circuits and Systems Part I, vol. 53, no. 3, pp. 712 721, Mar. 2006

[7] L. E. Larson, J-M. Liu, L. S. Tsimring, Digital Communications Using Chaos and Nonlinear Dynamics, Springer, 2006.

[8] S. Arai, Y. Nishio and T. Yamazato, "Error-Correcting Method Based on Chaotic Dynamics for Noncoherent Chaos Communications," ProcProc. NOLTA'08, pp. 652-655, Sep. 2008.

[9] S. Arai and Y. Nishio, "Suboptimal Receiver Using Shortest Distance Approximation Method for Chaos Shift Keying," RISP J. Signal Processing, vol. 13, no. 2, pp. 161-169, Mar. 2009.

[10] S. Arai, Y. Nishio and T. Yamazato, "No Redundant Error-Correcting Scheme Using Chaotic Dynamics for Noncoherent Chaos Communications," Proc. ISCAS'09, pp. 2633-2636, May 2009.

Fig. 7. Accuracy rate of error correction. 